\title{
Some Highlights of the URSI Symposium on Electromag- netic Wave Theory Held in Delft, The Netherlands, September 6-11, 1965
}

\author{
James R. Wait \\ Central Radio Propagation Laboratory, Environmental Science Services Administration, Boulder, Colo.
}

(Received October 5, 1965)

\section{The Scope of the Symposium}

Under the auspices of the International Scientific Radio Union, a symposium on electromagnetic wave theory was held at the Technological University in Delft, Holland. The organizing and technical committee was under the chairmanship of Dr. F. L. Stumpers and the secretary was Dr. R. Timman. Other members of the committee were Professor H. M. Barlow (UK), Dr. P. Grivet (France), Professor O. E. H. Rydbeck (Sweden), Professor K. M. Siegel (USA), Academician V. I. Siforov (USSR), Professor G. Toraldo di Francia (Italy), and Admiral H. A. W. Goossens, Professor Dr. A. T. de Hoop, Dr. J. P. Schouten, Dr. B. D. H. Tellegen, all of the Netherlands.

Actually, this symposium in Delft is a fifth in a quasi-periodic sequence. The predecessors were the Symposium on Microwave Optics at Montreal in 1953, the URSI Symposia on Electromagnetic Theory at Michigan in 1955; at Toronto in 1959, and at Copenhagen in 1962.

The scientific program was grouped under the following subjects, which were announced in advance of submission of papers:

Group 1 Propagation in inhomogeneous media

2 Antenna theory

3 Propagation in plasmas

4 Underground waves-space waves

5 Boundary value problems

6 Millimeter waves and optical waves Propagation in nonlinear media

8 Surface waves and wave beams

9 Coherence problems and modern optics (including wave front and statistical optics)

10 Multiple scattering

11 Deterministic scattering

The papers which finally appeared on the program, by and large, could be fitted into the groups indicated above. However, it was evident that papers dealing with the classical aspects (e.g., Groups 2 and 5) received the most attention. In this connection, one

1 J. B. Keller (1963), A survey of short wavelength diffraction theory, International Series of Monographs on Electromagnetic Waves, 6, 3-9 (Pergamon Press). wonders if many authors heeded the recommendation of J. B. Keller in his 1962 Copenhagen paper ${ }^{1}$ which is worth recording here: "It is essential that we infer general principles from all our results, and organize the results in terms of them. Otherwise our subject will degenerate into a collection of special solutions. This tendency is becoming especially ominous as computing machines invade diffraction theory."

While many papers were presentations of "special solutions," there certainly were notable attempts by some authors to draw general conclusions from their analysis.

In this present report of the conference, we shall identify a number of the papers which appear to provide new insight into the behavior of electromagnetic waves. Space limitations in this journal and the inability of this writer to be in attendance at both of the simultaneous sessions has resulted in the omission of mention here of other important papers presented at Delft.

\section{The Highlights}

Some major contributions to asymptotic diffraction theory were described by R. M. Lewis of New York University. In this class of problems, certain curves called rays, play an essential role. Their importance rests on the fact that the functions which are used to make up the solution actually satisfy ordinary differential equations in terms of the local coordinates of the ray system. This "ray method" has been studied extensively by J. B. Keller and his associates at the Courant Institute. Its principal advantage, of course, is that an acceptable asymptotic solution may be obtained without having to first find an exact solution. R. M. Lewis was ready to admit that there is no general proof that the ray method actually yields the asymptotic expansion of the exact solution. However, overwhelming evidence in support of the ray method is obtained by comparing the leading term of the asymptotic solution of the exact solution and the "ray solution" for particular problems. As an example, Lewis and his colleague N. Bleistein consider initial boundary problems in two space dimensions for the Klein-Gordon equation. In their problem, the 
boundary was a parabola and they required that the solution satisfied a homogeneous boundary condition. The total solution was assumed to consist of the sum of a primary and a reflected field. The associated primary and secondary rays are straight lines in spacetime. The primary field, of course, must satisfy the pertinent wave equation and the initial conditions. On the other hand, the reflected field is taken to be zero initially and is determined at the boundary in such a way that the total solution satisfies the given boundary condition. Using this "ray method," the leading terms of the primary and the reflected fields are then calculated. For a number of cases, these solutions are then compared with the exact results where they are available. The agreement is excellent for large values of a characteristic parameter.

The "ray method" mentioned was also used by Lewis to study the propagation of transients in dispersive media. His primary rays represented the trajectory (in space-time) of a point which moved with an appropriately defined group velocity. On the other hand, his transient rays corresponded to the second term of the asymptotic expansion of the solution. It is interesting to note that some regions of space-time are only reached by the transient rays. These correspond to the "precursors" considered originally by Sommerfeld and Brillouin in a different setting.

Another notable example of generalized ray concepts was covered in an excellent paper by L. B. Felsen of the United States. He indicated that the lateral waves associated with an interface between dissimilar media could be nicely characterized by a glancing ray. Again, the validity of the procedure could be checked by comparison with the exact solution of an appropriate canonical problem.

The impulse response of a surface wave structure was discussed by C. Kooy of the Netherlands. Using a rather ingenious method, he calculated the distortion of a $\delta$-impulse function as it propagated along a grounded dielectric slab in the form of a TM surface wave. The trick was to introduce a special variable in place of the usual complex frequency in the inverse Laplace integral. The resulting integral, while appearing more complicated, could yield readily to a functiontheoretic approach. The complete temporal behavior of the propagated waveform was then described in terms of the saddle points of the integral.

An alternative approach to the solution of a class of Wiener-Hopf and related problems was presented by R. Mittra and C. P. Bates of the United States. They showed that a formulation in terms of an infinite set of simultaneous equations could be handled by a direct function-theoretic approach rather than resorting to the formal factorization methods. An example which they mentioned was the problem of the step discontinuity in a waveguide. They also showed that their method will work even in aperiodic, open or semiinfinite geometries such as a parallel-plate waveguide radiating into space.

Turning to a slightly more practical view, Professor H. M. Barlow of the United Kingdom surveyed "Millimetre waves and optical waves for long distance tele- communication by waveguide." He called attention to the recent success in the UK with the circular $\mathrm{H}_{01}$ guide employing a dielectric lined copper tube for nominally straight lengths with helix fillers inserted at intervals along it and permitting deliberate bends by employing flexible corrugated brass tubes. These flexible lengths have an insertion loss of only $0.2 \mathrm{~dB}$ in the band from 30 to $40 \mathrm{Gc} / \mathrm{s}$ for bends from $0^{\circ}$ to $90^{\circ}$ with a radius of curvature of $10 \mathrm{~cm}$. The considerations that have led to the success of the British development in this field are worth noting here. The principal factor was the judicious choice of parameters which emphasized the breaking of degeneracy between the wanted and the unwanted wave modes at bends. Other pertinent factors mentioned by Dr. H. G. Effemey were: (i) The selection of $8 \mathrm{~mm}$ band rather than the shorter one used in the United States and Japan, (ii) recognition that the dielectric smooth bore copper tube with short helical wire fillers (introduced at intervals) are more economical than the continuous helix waveguide, and (iii) the use of a form of circularly corrugated tubes of smaller diameter than the main guide which provides for sharp bends by having a special circumferential variation of the surface impedance.

Professor Barlow also described some interesting applications of surface waves to the radar control of trains which are already in use in Japan. This development was an interesting application of the principle that a surface wave will not radiate until the uniformity of the guiding structure is disturbed.

The theory of propagation of waves in horns and through horn junctions was discussed in a very provocative paper by Dr. H. G. Unger of Germany. The success claimed for the method was attributed to the use of the natural coordinate system employed. For example, for sectoral horns, these are local cylindrical coordinate systems, while for round or conical horns, local spherical coordinates are used. By substituting the appropriate field expansions into Maxwell's expansions, Unger obtains an infinite system of linear first-order differential equations for the expansion coefficients. Thus, the general horn may be represented by an infinite number of mutually coupled nonuniform transmission lines. In contrast to previous analyses which did not use the "natural" coordinate systems, the convergence of the series solutions is very good. However, he found that for horns of varying cone angle, there was a significant interaction between all modes of equal circumferential order traveling in both directions. Unger also applied his results to the design of circular electric waveguide tapers with low mode conversion.

At the 1962 Copenhagen symposium, H. Meinke ${ }^{2}$ gave a comprehensive survey of the use of conformal transformations for solving waveguide problems. His method has been exploited by G. Flachenecker and K. Lange of Germany who, in Delft, presented a striking demonstration of the time and spatial dependence of the power flow in a rectangular waveguide

${ }^{2} \mathrm{H}$. Meinke, A survey on the use of conformal mapping for solving wave field problems, ibid, 1113-1124. 
with a half-round obstacle. The calculation procedure is straightforward since the configuration in the original problem is transformed to a parallel-plate waveguide which is loaded with inhomogeneous and isotropic media.

Diffraction by an aperture in an anisotropic medium was considered by E. V. Jull of Canada. He started with the solution of the half-plane problem under the restriction that the $\mathrm{d}-\mathrm{c}$ magnetic field was parallel to the edge. He then developed his results asymptotically which permitted a generalization to two half planes whose edges were parallel to one another (i.e., a slit). His approach was closely akin to the "ray method" of Keller. While Jull's results seemed entirely plausible, confirmation of his procedure might have been obtained if he had compared his results with an exact solution. The latter would be expressible in a poorly converging series of Mathieu functions. A closely related diffraction problem was discussed by N. R. Zitron of Denmark. He included higher order interactions between the two edges of the slit but, again, he did not compare his results with an exact solution.

A number of papers relating to electromagnetic interactions with plasma were presented at Delft. A notable example was a paper by H. J. Schmitt and G. Meltz of the United States. The theory, which they developed, explained the role of electroacoustic waves in electromagnetic scattering from warm gyrotropic plasmas. The analysis was based on the linearized Vlasov equation for wave motion, in a stratified plasma, perpendicular to the static magnetic field. A related nonlinear plasma problem was treated by J. L. Yen of Canada. Using a hydrodynamic description, he calculated the nonlinear scattering from a homogeneous warm plasma cylinder. The results provided a "partial explanation" for observed harmonic scattering when a high power beam is incident on an inhomogeneous hot plasma.

The general subject of antennas immersed in plasma received considerable attention at the 1962 symposium at Copenhagen. A number of papers on the same topic also appeared on the program for the Delft conference. A rather significant contribution was communicated (but not presented) by M. Katzin of the United States. He analyzed the radiation from a slotted cone surrounded by a conical plasma sheath. The results from this investigation would appear to shed considerable light on the strong effects observed when a space vehicle re-enters the atmosphere. To simplify the problem, a bit, he assumed that the ionization density, within the shock formed sheath, was constant. The fields were represented as an ingegral form at the outset. This permitted an arbitrary excitation of the slot in the surface of the radiating cone. He indicated, in his written summary, that the analysis may be extended to inhomogeneous sheaths corresponding to a tapering off of the ionization density into the wake region.

As usual in symposia of this kind, there were a large number of papers dealing with antenna theory in its purer form. Specific topics were: theory of Van Atta reflectors, radiation from a paraboloid of revolution, equiangular and conical spiral antennas, spherical omnidirectional antennas, uniform and nonuniform arrays of point sources, "backfire" antennas, and focussed apertures.

A particularly interesting antenna paper was given by J. Bach Andersen of Denmark who described a design procedure for surface wave antennas when both the source and the finite length of the guiding structure are taken into account. While the method was approximate, he found good agreement with exact solutions when the method was applied to various simpler problems. Although not specifically mentioned by Bach Andersen, the techniques are closely related to techniques used in solving mixed-path propagation problems.

\section{Final Remarks}

The symposium was highly successful in that it brought together electromagnetic theorists from many countries. During a midweek interlude, the delegates visited the "Delta" project. This involved a 4-hr boat trip which provided a wonderful opportunity to discuss questions not resolved at the sessions. A very enjoyable banquet was held at the "Kurhaus" in Scheveningen. The banquet speaker, Dr. Casimir of N. V. Philips, told a humorous fairy tale about electrical units.

The proceedings of the conference will be edited by Professor J. Brown, University College, London and will be published by the Pergamon Press.

(Paper 69D12-624) 